\title{
On the Quintic Nonlinear Schrödinger Equation Created by the Vibrations of a Square Plate on a Weakly Nonlinear Elastic Foundation and the Stability of the Uniform Solution
}

\author{
Ben T. Nohara* and Akio Arimoto ${ }^{\dagger}$ \\ * Department of Electronic and Computer Engineering, \\ Tamazutsumi, Setagaya, Tokyo 158-855\%, Japan \\ E-mail:drben@sc.musashi-tech.ac.jp \\ $\dagger$ Department of Mathematics, Musashi Institute of Technology, \\ Tamazutsumi, Setagaya, Tokyo 158-8557, Japan
}

Received March 3, 2006

Revised October 20, 2006

\begin{abstract}
Plates are common structural elements of most engineering structures, including aerospace, automotive, and civil engineering structures. The study of plates from theoretical perspective as well as experimental viewpoint is fundamental to understanding of the behavior of such structures. The dynamic characteristics of plates, such as natural vibrations, transient responses for the external forces and so on, are especially of importance in actual environments. In this paper, we conside the envelope surface created by the vibrations of a square plate on a weakly nonliner elastic foundation and analyze the stability of the uniform solution of the governing equation for the envelope surface. We derive the two-dimensional equation that governs the spatial and temporal evolution of the envelope surface on cubic nonlinear elastic foundation. The fact that the governing equation becomes the quintic nonlinear Schrödinger equation is shown. Also we obtain the stability condition of the uniform solution of the quintic nonlinear Schrödinger equation.
\end{abstract}

Key words: elastic foundation, envelope, nearly monochromatic waves, perturbation, Schrödinger equation

\section{Introduction}

Plates are common structural elements of most engineering structures, including aerospace, automotive, and civil engineering structures. The study of plates from theoretical perspective as well as experimental viewpoint is fundamental to understanding of the behavior of such structures. The dynamic characteristics of plates, such as natural vibrations, transient responses for the external forces and so on, are especially of importance in actual environments. In this paper, we conside the envelope surface created by the vibrations of a square plate on a weakly nonliner elastic foundation and analyze the stability of the uniform solution of the governing equation for the envelope surface. We derive the two-dimensional equation that governs the spatial and temporal evolution of the envelope surface and discuss the stability of the uniform solution.

In the course of studying the theory of plates the classical, Kirchhoff plate theory [1]-[3], in which transverse normal and shear stresses are neglected to study bending, buckling, and natural vibrations of rectangular plates, was first established. The treatment of the linear vibrations of plates is comprehensively given in 
the monograph [4]. The governing equations of the nonlinear vibrations of plates was also reduced [5]. The first-order shear deformation plate theory extends the kinematics of the classical, Kirchhoff plate theory by relaxing the normality restriction and allowing for arbitrary but constant rotation of transverse normals $[6,7]$ and finite element models are developed for the precise analysis of the plate characteristics in real problems [8].

In general, the Schrödinger equation [9] governs the spatial and temporal evolution of the amplitude of a wavepacket propagating transversely in any dispersive, lossless medium. The spatial and temporal evolution of the amplitude of a wavepacket centered around a wavenumber and a frequency is varying slowly in space and time so that it creates an envelope. In other words, the Schrödinger equation governs an envelope created by a wavepacket. The nonlinear Schrödinger equation arises in the nonlinear dispersive characteristics of propagation medium and nonlinear restoring force and so on. Many studies of a wavepacket has been carried out in water wave [10]-[12], plasma [13], fiber-optic communication systems [14], and some other area as well. Moreover, several Schrödinger type equations are derived from the wavenumber-based or directional-based spectrum of nearly monochromatice waves and their stabilities of the solutions are analyzed [15, 16]. Nearly bichromatic waves which are expanded from nearly monochromatice waves are also analyzed and the related equations govern envelopes created by nearly bichromatic waves are derived. The nonlinear dynamics and numerical simulations of its solutions are performed [17]-[20].

We can consider the force due to the elastic foundation is propotional to the second or third power of the displacement. The past studies have only treated the second power of the displacement [21]. However, when considering the nonlinearity of an elastic foundation, the third power of the displacement is naturally considered. In this paper, we derive the two-dimensional governing equation that describes the propagation of the envelope surface of a square plate on an elastic foundation using the method of multiple scales [22]. The obtained equation becomes the quintic nonlinear Schrödinger equation. We consider the stability of the uniform solution of the obtained quintic nonlinear Schrödinger equation through the modulational perturbation. The sufficient condition of the stability is shown.

The following section presents the plate equation on an elastic, weakly nonlinear foundation with cubic nonlinearity. In the third section we derive the govrning equation of the envelope surface of nearly monochromatic waves on cubic nonlinear elastic foundation using the method of multiple scales. In the fourth section we analyze the stability of the uniform solution of the obtained governing equation in the previous section.

\section{Preliminary: Plate equation on an elastic, weakly nonlinear founda- tion [21]}

We consider a square plate with side length $l$. The mass of the plate per unit area perpendicular to $z$-axis, the mass density of the plate, the area of the cross 
sectoin of the plate perpendicular to $x$-axis, the elasticity modulus, and the moment of inertia of the cross section with respect to the $x$-axis are denoted by $\mu, \rho, A_{a}$, $E$, and $I$, respectively. Moreover, the weight $W$ of the plate per unit area is set to be constant, that is, $W=\mu g$ ( $g$ is the gravitational acceleration) and we neglect internal damping. Then, the equation of motion for the vertical displacement of the plate $w(x, y, t)$, in which $t$ is time, is given by

$$
\begin{aligned}
& \mu \frac{\partial^{2} w(x, y, t)}{\partial t^{2}}+E I\left\{\frac{\partial^{4} w(x, y, t)}{\partial x^{4}}+2 \frac{\partial^{4} w(x, y, t)}{\partial x^{2} \partial y^{2}}+\frac{\partial^{4} w(x, y, t)}{\partial y^{4}}\right\} \\
& +F(w(x, y, t))=-\mu g, \\
& 0<x<l, \quad 0<y<l, \quad t>0
\end{aligned}
$$

where $F$ is the force in $z$-direction per unit area acting on the plate due to the elastic foundation. We consider the free oscillations of the plate without specific boundary conditions.

We assume that the vertical displacements of the plate are small compared to the length $l$. We also assume that the force $F(w)$ can be naturally written as follows:

$$
F(w)=k_{s} w+b_{s} w^{3}
$$

where $k_{s}$ and $b_{s}$ are spring constants. Constant $k_{s}$ must be positive physically. Constant $b_{s}$ takes either zero or positive or negative. If $b_{s}$ is zero, then the spring is a linear spring. For a linear spring, the force is proportional to the displacement. If $b_{s}$ is not zero, then the spring is a nonlinear spring [23]. If $b_{s}$ is positive (we call a hard spring), then the nonlinearity increases the force. If $b_{s}$ is negative (we call a soft spring), then the nonlinearity decreases the force. We are interested in a nonlinear spring, that is, $b_{s}$ is positive or negative. In the appendix, the governing equation with a nonlinear spring expressed by

$$
F(w)=k_{s} w+b_{s} w^{2}
$$

is shown. In this case, we have the known result [21].

Then Equation (1) becomes

$$
\begin{aligned}
& \frac{\partial^{2} w(x, y, t)}{\partial t^{2}}+\frac{E I}{\mu}\left\{\frac{\partial^{4} w(x, y, t)}{\partial x^{4}}+2 \frac{\partial^{4} w(x, y, t)}{\partial x^{2} \partial y^{2}}+\frac{\partial^{4} w(x, y, t)}{\partial y^{4}}\right\} \\
& +\frac{k_{s}}{\mu} w(x, y, t)+\frac{b_{s}}{\mu} w(x, y, t)^{3}=-g .
\end{aligned}
$$

In order to simplify equation (4) the term $-g$ will be removed by introducing the transformation

$$
w(x, y, t)=\tilde{w}(x, y, t)+\frac{\mu g}{k_{s}} s(x, y)
$$


where $s(x, y)$ satisfies the following time-independent equation:

$$
\frac{\partial^{4} s(x, y)}{\partial x^{4}}+2 \frac{\partial^{4} s(x, y)}{\partial x^{2} \partial y^{2}}+\frac{\partial^{4} s(x, y)}{\partial y^{4}}+\frac{k_{s}}{E I} s(x, y)=-\frac{k_{s}}{E I},
$$

where $\frac{\mu g}{k_{s}} s(x, y)$ represents the deflection of the plate in static state due to gravity. Equation (6) is easily solved as the boundary value problem, but we are not interested in static state. $\tilde{w}(x, y, t)$ is written as follows:

$$
\begin{gathered}
\frac{\partial^{2} \tilde{w}(x, y, t)}{\partial t^{2}}+\frac{E I}{\mu}\left\{\frac{\partial^{4} \tilde{w}(x, y, t)}{\partial x^{4}}+2 \frac{\partial^{4} \tilde{w}(x, y, t)}{\partial x^{2} \partial y^{2}}+\frac{\partial^{4} \tilde{w}(x, y, t)}{\partial y^{4}}\right\}+\frac{k_{s}}{\mu} \tilde{w}(x, y, t) \\
+\frac{b_{s}}{\mu}\left\{\tilde{w}(x, y, t)^{3}+\frac{3 \mu g}{k_{s}} \tilde{w}(x, y, t)^{2} s(x, y)\right. \\
\left.\quad+3\left(\frac{\mu g}{k_{s}}\right)^{2} \tilde{w}(x, y, t) s(x, y)^{2}+\left(\frac{\mu g}{k_{s}}\right)^{3} s(x, y)^{3}\right\}=0 .
\end{gathered}
$$

Using the dimensionless variables

$$
\bar{w}=\frac{l}{A_{a}} \tilde{w}, \quad \bar{x}=\frac{\pi}{l} x, \quad \bar{y}=\frac{\pi}{l} y, \quad \bar{t}=\left(\frac{\pi}{l}\right)^{2} \sqrt{\frac{E I}{\mu}} t,
$$

equation (7) becomes

$$
\begin{aligned}
& \frac{\partial^{2} \bar{w}}{\partial \bar{t}^{2}}+\frac{\partial^{4} \bar{w}}{\partial \bar{x}^{4}}+2 \frac{\partial^{4} \bar{w}}{\partial \bar{x}^{2} \partial \bar{y}^{2}}+\frac{\partial^{4} \bar{w}}{\partial \bar{y}^{4}} \\
& +\frac{l^{4}}{\pi^{4} E I}\left\{k_{s} \bar{w}+\frac{b_{s} A_{a}^{2}}{l^{2}} \bar{w}^{3}+\frac{3 b_{s} A_{a}}{l} \bar{w}^{2} \frac{\mu g}{k_{s}} s\left(\frac{l}{\pi} \bar{x}, \frac{l}{\pi} \bar{y}\right)\right. \\
& \left.\quad+\frac{b_{s} A_{a}^{2}}{l^{2}} \bar{w}\left(\frac{\mu g}{k_{s}} s\left(\frac{l}{\pi} \bar{x}, \frac{l}{\pi} \bar{y}\right)\right)^{2}+\frac{b_{s} l}{A_{a}}\left(\frac{\mu g}{k_{s}} s\left(\frac{l}{\pi} \bar{x}, \frac{l}{\pi} \bar{y}\right)\right)^{3}\right\}=0
\end{aligned}
$$

where we simply write $\bar{w}$, which is $\bar{w}\left(\frac{l}{\pi} \bar{x}, \frac{l}{\pi} \bar{y},\left(\frac{l}{\pi}\right)^{2} \sqrt{\frac{\mu}{E I}} \bar{t}\right)$ exactly.

We assume that the area $A_{a}$ of the cross section is small compared to the plate side length $l$, then we put $\tilde{\varepsilon}=\left(\frac{A_{a}}{l}\right)^{2}$ with $\tilde{\varepsilon}$ a small parameter. We also assume that the deflection of the plate in static state due to gravity, $\frac{\mu g}{k_{s}} s(x, y)$, is small with respect to the vertical displacement $\tilde{w}$. So, we assume that $\frac{\mu g}{k_{s}} s(x, y)$ is $\mathcal{O}\left(\tilde{\varepsilon}^{n}\right)$ with $n>1$. Setting

$$
\varepsilon=-b_{s} \tilde{\varepsilon}\left(\frac{l}{\pi}\right)^{4} \frac{1}{E I}, \quad p^{2}=\left(\frac{l}{\pi}\right)^{4} \frac{k_{s}}{E I},
$$

equation (9) becomes

$$
\frac{\partial^{2} \bar{w}}{\partial \bar{t}^{2}}+\frac{\partial^{4} \bar{w}}{\partial \bar{x}^{4}}+2 \frac{\partial^{4} \bar{w}}{\partial \bar{x}^{2} \partial \bar{y}^{2}}+\frac{\partial^{4} \bar{w}}{\partial \bar{y}^{4}}+p^{2} \bar{w}=\varepsilon \bar{w}^{3}+\mathcal{O}\left(\varepsilon^{n}\right),
$$


with $n>1$ and $\varepsilon$ is a small parameter. We can now write the following equation governs the vertical displacement of a plate on a weakly nonlinear, elastic foundation, which describes up to $\mathcal{O}\left(\varepsilon^{n}\right), n>1$ :

$$
\begin{aligned}
& \frac{\partial^{2} w(x, y, t)}{\partial t^{2}}+\frac{\partial^{4} w(x, y, t)}{\partial x^{4}}+2 \frac{\partial^{4} w(x, y, t)}{\partial x^{2} \partial y^{2}}+\frac{\partial^{4} w(x, y, t)}{\partial y^{4}}+p^{2} w(x, y, t) \\
& =\varepsilon w(x, y, t)^{3} \\
& 0<x<\pi, \quad 0<y<\pi, \quad t>0,
\end{aligned}
$$

where we drop all bars for convenience. The first four terms in the left-hand side of equation (12) are the linear part of the plate equation and $p^{2} w-\varepsilon w^{3}$ represents the restoring force due to the elastic foundation. Since no other external forces are considered, equation (12) describes the free osillations on an elastic, weakly nonlinear foundation.

\section{Governing equation of the envelope surface of nearly monochromatic waves on an elastic, weakly nonlinear foundation}

In this section we derive the governing equation for the envelope surface created by nearly monochromatic waves propagating in unidirection on an plate with weakly nonlinear foundation. Nearly monochromatic waves have the wavenumber spectrum having a peak and spreading over around a peak. So, the energy of nearly monochromatic waves is almost concentrated in a single wavenumber. The amplitude of such waves varies slowly in time and space so that creates the envelope of traveling waves.

In order to derivate the equation govern the envelope of nearly monochromatic waves centered around the wavenumber $k_{w}$ and the angular frequency $\omega$, we use the method of multiple scales [22]. We introduce the slow time scales $T_{1}=\epsilon t$, and $T_{2}=\epsilon^{2} t$ in addition to the original time scale $T_{0}=t$. Moreover we introduce the long scales $X_{1}=\epsilon x, X_{2}=\epsilon^{2} x, Y_{1}=\epsilon y$, and $Y_{2}=\epsilon^{2} y$ in addition to the original space scale $X_{0}=x$ and $Y_{0}=y$. Here $\epsilon$ is a small parameter, which is physically different from $\varepsilon$ in equation (10). Both $\epsilon$ and $\varepsilon$ are the same small parameter mathematically and we assume that $\epsilon$ and $\varepsilon$ are of the same order, so we denote $\epsilon$ without distinction. Hence the time and space derivatives become

$$
\begin{aligned}
\frac{\partial}{\partial t} & =\frac{\partial}{\partial T_{0}}+\epsilon \frac{\partial}{\partial T_{1}}+\epsilon^{2} \frac{\partial}{\partial T_{2}}, \\
\frac{\partial}{\partial x} & =\frac{\partial}{\partial X_{0}}+\epsilon \frac{\partial}{\partial X_{1}}+\epsilon^{2} \frac{\partial}{\partial X_{2}}, \\
\frac{\partial}{\partial y} & =\frac{\partial}{\partial Y_{0}}+\epsilon \frac{\partial}{\partial Y_{1}}+\epsilon^{2} \frac{\partial}{\partial Y_{2}} .
\end{aligned}
$$

Then we seek a second-order solution in the form

$$
w(x, y, t ; \epsilon)=\sum_{n=0}^{2} \epsilon^{n} w_{n}\left(X_{0}, X_{1}, X_{2}, Y_{0}, Y_{1}, Y_{2}, T_{0}, T_{1}, T_{2}\right)+\mathcal{O}\left(\epsilon^{3}\right) .
$$


Substituting equation (14) into equation (12), using equation (13), and equating coefficients of like powers of $\epsilon$, we obtain

$$
\begin{aligned}
& \frac{\partial^{4} w_{0}}{\partial X_{0}^{4}}+2 \frac{\partial^{4} w_{0}}{\partial X_{0}^{2} \partial Y_{0}^{2}}+\frac{\partial^{4} w_{0}}{\partial Y_{0}^{4}}+\frac{\partial^{2} w_{0}}{\partial T_{0}^{2}}+p^{2} w_{0}=0 \\
& \frac{\partial^{4} w_{1}}{\partial X_{0}^{4}}+2 \frac{\partial^{4} w_{1}}{\partial X_{0}^{2} \partial Y_{0}^{2}}+\frac{\partial^{4} w_{1}}{\partial Y_{0}^{4}}+\frac{\partial^{2} w_{1}}{\partial T_{0}^{2}}+p^{2} w_{1} \\
& =-4 \frac{\partial^{4} w_{0}}{\partial X_{0}^{3} \partial X_{1}}-4 \frac{\partial^{4} w_{0}}{\partial X_{0} \partial X_{1} \partial Y_{0}^{2}}-4 \frac{\partial^{4} w_{0}}{\partial X_{0}^{2} \partial Y_{0} \partial Y_{1}} \\
& \quad-4 \frac{\partial^{4} w_{0}}{\partial Y_{0}^{3} \partial Y_{1}}-2 \frac{\partial^{2} w_{0}}{\partial T_{0} \partial T_{1}}+w_{0}^{3} \\
& \frac{\partial^{4} w_{2}}{\partial X_{0}^{4}}+2 \frac{\partial^{4} w_{2}}{\partial X_{0}^{2} \partial Y_{0}^{2}}+\frac{\partial^{4} w_{2}}{\partial Y_{0}^{4}}+\frac{\partial^{2} w_{2}}{\partial T_{0}^{2}}+p^{2} w_{2} \\
& =-4 \frac{\partial^{4} w_{1}}{\partial X_{0}^{3} \partial X_{1}}-4 \frac{\partial^{4} w_{1}}{\partial X_{0} \partial X_{1} \partial Y_{0}^{2}}-4 \frac{\partial^{4} w_{1}}{\partial X_{0}^{2} \partial Y_{0} \partial Y_{1}} \\
& \quad-4 \frac{\partial^{4} w_{1}}{\partial Y_{0}^{3} \partial Y_{1}}-2 \frac{\partial^{2} w_{1}}{\partial T_{0} \partial T_{1}}-6 \frac{\partial^{4} w_{0}}{\partial X_{0}^{2} \partial X_{1}^{2}}-4 \frac{\partial^{4} w_{0}}{\partial X_{0}^{3} \partial X_{2}}-8 \frac{\partial^{4}}{\partial X_{0} \partial X_{1} \partial Y_{0} \partial Y_{1}} \\
& \quad-2 \frac{\partial^{4} w_{0}}{\partial X_{1}^{2} \partial Y_{0}^{2}}-4 \frac{\partial^{4} w_{0}}{\partial X_{0} \partial X_{2} \partial Y_{0}^{2}}-2 \frac{\partial^{4} w_{0}}{\partial X_{0}^{2} \partial Y_{1}^{2}}-4 \frac{\partial^{4} w_{0}}{\partial X_{0}^{2} \partial Y_{0} \partial Y_{2}} \\
& \quad-6 \frac{\partial^{4} w_{0}}{\partial Y_{0}^{2} \partial Y_{1}^{2}}-4 \frac{\partial^{4} w_{0}}{\partial Y_{0}^{3} \partial Y_{2}}-\frac{\partial^{2} w_{0}}{\partial T_{1}^{2}}-2 \frac{\partial^{2} w_{0}}{\partial T_{0} \partial T_{2}}+3 w_{0}^{2} w_{1} \cdot
\end{aligned}
$$

To analyze the propagation of nearly monochromatic waves centered around the wavenumber $k_{w}$ and the angular frequency $\omega$, we take the solution of equation (15) in the form

$$
\begin{aligned}
w_{0}= & A\left(X_{1}, X_{2}, Y_{1}, Y_{2}, T_{1}, T_{2}\right) e^{i\left(k_{w} X_{0} \cos \theta_{0}+k_{w} Y_{0} \sin \theta_{0}-\omega T_{0}\right)} \\
& +A^{*}\left(X_{1}, X_{2}, Y_{1}, Y_{2}, T_{1}, T_{2}\right) e^{-i\left(k_{w} X_{0} \cos \theta_{0}+k_{w} Y_{0} \sin \theta_{0}-\omega T_{0}\right)} .
\end{aligned}
$$

We write this equation as follows for convenience (hereinafter, "cc" is used as the same manner):

$$
w_{0}=A\left(X_{1}, X_{2}, Y_{1}, Y_{2}, T_{1}, T_{2}\right) e^{i\left(k_{w} X_{0} \cos \theta_{0}+k_{w} Y_{0} \sin \theta_{0}-\omega T_{0}\right)}+\mathrm{cc} .
$$

Equation (18) describes the propagation of nearly monochromatic waves whose propagation direction is $\theta_{0}\left(0<\theta_{0}<\pi / 2\right)$ in the $(x, y)$ plane. In other words, $A$ of equation (18) presents the envelope surface created by nearly monochromatic waves. We derive the governing equation for $A$ using equations (15), (16), (17), and (18).

First, the dispersion relation for $A$ is led by substituting equation (18) into equation (15)

$$
\omega^{2}=k_{w}^{4}+p^{2} .
$$


Next, substituting equation (18) into equation (16) and eliminating the terms that produce secular terms yield the following solvability condition

$$
4 i k_{w}^{3}\left(\cos \theta_{0} \frac{\partial A}{\partial X_{1}}+\sin \theta_{0} \frac{\partial A}{\partial Y_{1}}\right)+2 i \omega \frac{\partial A}{\partial T_{1}}+3|A|^{2} A=0
$$

and we have the equation $w_{1}$ satisfies as follows:

$$
\frac{\partial^{4} w_{1}}{\partial X_{0}^{4}}+2 \frac{\partial^{4} w_{1}}{\partial X_{0}^{2} \partial Y_{0}^{2}}+\frac{\partial^{4} w_{1}}{\partial Y_{0}^{4}}+\frac{\partial^{2} w_{1}}{\partial T_{0}^{2}}+p^{2} w_{1}=A^{3} e^{3 i\left(k_{w} X_{0} \cos \theta_{0}+k_{w} Y_{0} \sin \theta_{0}-\omega T_{0}\right)}+c c .
$$

Then the solution of equation (21) becomes

$$
w_{1}=\frac{A^{3}}{8\left(9 k_{w}^{4}-p^{2}\right)} e^{3 i\left(k_{w} X_{0} \cos \theta_{0}+k_{w} Y_{0} \sin \theta_{0}-\omega T_{0}\right)}+\mathrm{cc} .
$$

Similarly, we eliminate the terms that produce secular terms in the equation obtained from equations (17), (18), and (22), then we obtain the solvability condition as follows:

$$
\begin{aligned}
& \left(6 k_{w}^{2} \cos ^{2} \theta_{0}+2 k_{w}^{2} \sin ^{2} \theta_{0}\right) \frac{\partial^{2} A}{\partial X_{1}^{2}}+4 i k_{w}^{3} \cos \theta_{0} \frac{\partial A}{\partial X_{2}}+4 k_{w}^{2} \sin 2 \theta_{0} \frac{\partial^{2} A}{\partial X_{1} \partial Y_{1}} \\
& +\left(6 k_{w}^{2} \sin ^{2} \theta_{0}+2 k_{w}^{2} \cos ^{2} \theta_{0}\right) \frac{\partial^{2} A}{\partial Y_{1}^{2}}+4 i k_{w}^{3} \sin \theta_{0} \frac{\partial A}{\partial Y_{2}}+2 i \omega \frac{\partial A}{\partial T_{2}}-\frac{\partial^{2} A}{\partial T_{1}^{2}}=0
\end{aligned}
$$

Equations (20) and (23) describe the evolution of the complex amplitude $A$ with the slow and long scales. We obtain the governing equation for $A$, which is accomplished by combining equations (20) and (23). First, from equation (19), we obtain the following relations:

$$
\omega \omega^{\prime}=2 k_{w}^{3}, \quad \omega \omega^{\prime \prime}=6 k_{w}^{2}-\frac{4 k_{w}^{6}}{k_{w}^{4}+p^{2}},
$$

where $\omega^{\prime}=d \omega / d k_{w}$ and $\omega^{\prime \prime}=d^{2} \omega / d k_{w}^{2}$. We use these relations in the calculation below. Next, from equation (20), we have

$$
\frac{\partial A}{\partial T_{1}}=-2 \frac{k_{w}^{3}}{\omega}\left(\cos \theta_{0} \frac{\partial A}{\partial X_{1}}+\sin \theta_{0} \frac{\partial A}{\partial Y_{1}}\right)+\frac{i 3}{2 \omega}|A|^{2} A
$$

Differentiating both sides of equation (25) with respect to $T_{1}$ yields

$$
\begin{aligned}
\frac{\partial^{2} A}{\partial T_{1}^{2}} & =-\omega^{\prime}\left(\cos \theta_{0} \frac{\partial}{\partial X_{1}} \frac{\partial A}{\partial T_{1}}+\sin \theta_{0} \frac{\partial}{\partial Y_{1}} \frac{\partial A}{\partial T_{1}}\right)+\frac{i 3}{2 \omega}\left(\frac{\partial|A|^{2}}{\partial T_{1}} A+\frac{\partial A}{\partial T_{1}}|A|^{2}\right) \\
& =-\omega^{\prime}\left(\cos \theta_{0} \frac{\partial}{\partial X_{1}} \frac{\partial A}{\partial T_{1}}+\sin \theta_{0} \frac{\partial}{\partial Y_{1}} \frac{\partial A}{\partial T_{1}}\right)+\frac{i 3}{2 \omega}\left(2|A|^{2} \frac{\partial A}{\partial T_{1}}+\frac{\partial A^{*}}{\partial T_{1}} A^{2}\right) .
\end{aligned}
$$


Substituting equation (25) to equation (26) yields

$$
\begin{aligned}
\frac{\partial^{2} A}{\partial T_{1}^{2}}=-\omega^{\prime}\left[\cos \theta_{0} \frac{\partial}{\partial X_{1}}\left\{-\omega^{\prime}\left(\cos \theta_{0} \frac{\partial A}{\partial X_{1}}+\sin \theta_{0} \frac{\partial A}{\partial Y_{1}}\right)+\frac{i 3}{2 \omega}|A|^{2} A\right\}\right. \\
\left.\quad+\sin \theta_{0} \frac{\partial}{\partial Y_{1}}\left\{-\omega^{\prime}\left(\cos \theta_{0} \frac{\partial A}{\partial X_{1}}+\sin \theta_{0} \frac{\partial A}{\partial Y_{1}}\right)+\frac{i 3}{2 \omega}|A|^{2} A\right\}\right] \\
\quad+\frac{i 3}{2 \omega}\left[2|A|^{2}\left\{-\omega^{\prime}\left(\cos \theta_{0} \frac{\partial A}{\partial X_{1}}+\sin \theta_{0} \frac{\partial A}{\partial Y_{1}}\right)+\frac{i 3}{2 \omega}|A|^{2} A\right\}\right. \\
\left.\quad+A^{2}\left\{-\omega^{\prime}\left(\cos \theta_{0} \frac{\partial A^{*}}{\partial X_{1}}+\sin \theta_{0} \frac{\partial A^{*}}{\partial Y_{1}}\right)-\frac{i 3}{2 \omega}|A|^{2} A^{*}\right\}\right] \\
=\omega^{\prime 2}\left(\cos ^{2} \theta_{0} \frac{\partial^{2} A}{\partial X_{1}^{2}}+2 \cos \theta_{0} \sin \theta_{0} \frac{\partial^{2} A}{\partial X_{1} \partial Y_{1}}+\sin ^{2} \theta_{0} \frac{\partial^{2} A}{\partial Y_{1}^{2}}\right) \\
-\frac{i 6 \omega^{\prime}}{\omega}|A|^{2}\left(\cos \theta_{0} \frac{\partial A}{\partial X_{1}}+\sin \theta_{0} \frac{\partial A}{\partial Y_{1}}\right) \\
-\frac{i 3 \omega^{\prime}}{\omega} A^{2}\left(\cos \theta_{0} \frac{\partial A^{*}}{\partial X_{1}}+\sin \theta_{0} \frac{\partial A^{*}}{\partial Y_{1}}\right)-\frac{9}{4 \omega^{2}}|A|^{4} A .
\end{aligned}
$$

Substituting equation (27) into equation (23) and arranging terms leads to

$$
\begin{aligned}
& i\left\{\frac{\partial A}{\partial T_{2}}+\omega^{\prime}\left(\cos \theta_{0} \frac{\partial A}{\partial X_{2}}+\sin \theta_{0} \frac{\partial A}{\partial Y_{2}}\right)+\frac{3 \omega^{\prime}}{\omega^{2}}|A|^{2}\left(\cos \theta_{0} \frac{\partial A}{\partial X_{1}}+\sin \theta_{0} \frac{\partial A}{\partial Y_{1}}\right)\right. \\
& \left.\quad+\frac{3 \omega^{\prime}}{2 \omega^{2}} A^{2}\left(\cos \theta_{0} \frac{\partial A^{*}}{\partial X_{1}}+\sin \theta_{0} \frac{\partial A^{*}}{\partial Y_{1}}\right)\right\} \\
& +\left\{\frac{1}{2} \omega^{\prime \prime} \cos ^{2} \theta_{0}+\frac{1}{6}\left(\omega^{\prime \prime}+\frac{\omega^{\prime 2}}{\omega}\right) \sin ^{2} \theta_{0}\right\} \frac{\partial^{2} A}{\partial X_{1}^{2}} \\
& +\left\{\frac{1}{2} \omega^{\prime \prime} \sin ^{2} \theta_{0}+\frac{1}{6}\left(\omega^{\prime \prime}+\frac{\omega^{\prime 2}}{\omega}\right) \cos ^{2} \theta_{0}\right\} \frac{\partial^{2} A}{\partial Y_{1}^{2}}+\frac{1}{3}\left(\omega^{\prime \prime}-\frac{\omega^{\prime 2}}{2 \omega}\right) \sin 2 \theta_{0} \frac{\partial^{2} A}{\partial X_{1} \partial Y_{1}} \\
& +\frac{9}{8 \omega^{3}}|A|^{4} A=0 .
\end{aligned}
$$

Expressing $X_{1}, X_{2}, Y_{1}, Y_{2}$, and $T_{2}$ in terms of the original $x, y$, and $t$ variables, we obtain

$$
\begin{aligned}
& i\left\{\frac{\partial A}{\partial t}+\left(\omega^{\prime}+\frac{3 \epsilon \omega^{\prime}}{\omega^{2}}|A|^{2}\right)\left(\cos \theta_{0} \frac{\partial A}{\partial x}+\sin \theta_{0} \frac{\partial A}{\partial y}\right)\right. \\
& \left.+\frac{3 \epsilon \omega^{\prime}}{2 \omega^{2}} A^{2}\left(\cos \theta_{0} \frac{\partial A^{*}}{\partial x}+\sin \theta_{0} \frac{\partial A^{*}}{\partial y}\right)\right\} \\
& +\left\{\frac{1}{2} \omega^{\prime \prime} \cos ^{2} \theta_{0}+\frac{1}{6}\left(\omega^{\prime \prime}+\frac{\omega^{\prime 2}}{\omega}\right) \sin ^{2} \theta_{0}\right\} \frac{\partial^{2} A}{\partial x^{2}} \\
& +\left\{\frac{1}{2} \omega^{\prime \prime} \sin ^{2} \theta_{0}+\frac{1}{6}\left(\omega^{\prime \prime}+\frac{\omega^{\prime 2}}{\omega}\right) \cos ^{2} \theta_{0}\right\} \frac{\partial^{2} A}{\partial y^{2}} \\
& +\frac{1}{3}\left(\omega^{\prime \prime}-\frac{\omega^{\prime 2}}{2 \omega}\right) \sin 2 \theta_{0} \frac{\partial^{2} A}{\partial x \partial y}+\frac{9 \epsilon^{2}}{8 \omega^{3}}|A|^{4} A=0 .
\end{aligned}
$$


To eliminate the cross term of $\frac{\partial^{2}}{\partial x \partial y}$, we transform the coordinate system by

$$
\left(\begin{array}{l}
\tilde{x} \\
\tilde{y} \\
\tilde{t}
\end{array}\right)=\left(\begin{array}{ccc}
\sqrt{\beta} & -\sqrt{\alpha} & 0 \\
\sqrt{\beta} & \sqrt{\alpha} & 0 \\
0 & 0 & 1
\end{array}\right)\left(\begin{array}{l}
x \\
y \\
t
\end{array}\right),
$$

where $\alpha=\frac{1}{2} \omega^{\prime \prime} \cos ^{2} \theta_{0}+\frac{1}{6}\left(\omega^{\prime \prime}+\frac{\omega^{\prime 2}}{\omega}\right) \sin ^{2} \theta_{0}, \beta=\frac{1}{2} \omega^{\prime \prime} \sin ^{2} \theta_{0}+\frac{1}{6}\left(\omega^{\prime \prime}+\frac{\omega^{\prime 2}}{\omega}\right) \cos ^{2} \theta_{0}$. (Note that $\alpha>0, \beta>0$ are easily found using equation (24).) We also let $c_{1}=\frac{3 \omega^{\prime}}{\omega^{2}}$, $c_{2}=\frac{1}{2} c_{1}=\frac{3 \omega^{\prime}}{2 \omega^{2}}, c_{3}=\frac{9}{8 \omega^{3}}$, and $\gamma=\frac{1}{3}\left(\omega^{\prime \prime}-\frac{\omega^{\prime 2}}{2 \omega}\right) \sin 2 \theta_{0}$, then equation (29) is transformed to the new coordinate system $(\tilde{x}, \tilde{y}, \tilde{t})$ as follows:

$$
\begin{aligned}
& i\left\{\frac{\partial A}{\partial \tilde{t}}+\left(\omega^{\prime}+\epsilon c_{1}|A|^{2}\right)\left(\left(\sqrt{\beta} \cos \theta_{0}-\sqrt{\alpha} \sin \theta_{0}\right) \frac{\partial A}{\partial \tilde{x}}+\left(\sqrt{\beta} \cos \theta_{0}+\sqrt{\alpha} \sin \theta_{0}\right) \frac{\partial A}{\partial \tilde{y}}\right)\right. \\
& \left.\quad+\epsilon c_{2} A^{2}\left(\left(\sqrt{\beta} \cos \theta_{0}-\sqrt{\alpha} \sin \theta_{0}\right) \frac{\partial A^{*}}{\partial \tilde{x}}+\left(\sqrt{\beta} \cos \theta_{0}+\sqrt{\alpha} \sin \theta_{0}\right) \frac{\partial A^{*}}{\partial \tilde{y}}\right)\right\} \\
& +(2 \alpha \beta-\gamma \sqrt{\alpha \beta}) \frac{\partial^{2} A}{\partial \tilde{x}^{2}}+(2 \alpha \beta+\gamma \sqrt{\alpha \beta}) \frac{\partial^{2} A}{\partial \tilde{y}^{2}}+\epsilon^{2} c_{3}|A|^{4} A=0
\end{aligned}
$$

Hereinafter, we use anew $x, y$, and $t$ instead of $\tilde{x}, \tilde{y}$, and $\tilde{t}$ in order to avoid a nuisance of symbols and we introduce the following symbols:

$$
\begin{aligned}
& a=\sqrt{\beta} \cos \theta_{0}-\sqrt{\alpha} \sin \theta_{0}, \quad b=\sqrt{\beta} \cos \theta_{0}+\sqrt{\alpha} \sin \theta_{0}, \\
& c=2 \alpha \beta-\gamma \sqrt{\alpha \beta}, \quad d=2 \alpha \beta+\gamma \sqrt{\alpha \beta} .
\end{aligned}
$$

Summarizing this section, we obtain the governing equation of the envelope surface of nearly monochromatic waves on an elastic, weakly nonlinear foundation as follows:

$$
\begin{aligned}
& i\left\{\frac{\partial A}{\partial t}+\left(\omega^{\prime}+\epsilon c_{1}|A|^{2}\right)\left(a \frac{\partial A}{\partial x}+b \frac{\partial A}{\partial y}\right)+\epsilon c_{2} A^{2}\left(a \frac{\partial A^{*}}{\partial x}+b \frac{\partial A^{*}}{\partial y}\right)\right\} \\
& +c \frac{\partial^{2} A}{\partial x^{2}}+d \frac{\partial^{2} A}{\partial y^{2}}+\epsilon^{2} c_{3}|A|^{4} A=0 .
\end{aligned}
$$

We note that equation (33) is a quintic nonlinear Schrödinger equation.

\section{Stability analysis of the uniform solution}

In this section, we analyze the stability of the uniform solution of equation (33). We obtain the following uniform solution of equation (33):

$$
A(t)=A_{0} e^{i\left(\epsilon^{2} c_{3} A_{0}^{4} t+\beta_{0}\right)}
$$

where $A_{0}$ and $\beta_{0}$ are real constants. We consider a modulational perturbation [24] of equation (34) and express it in the form

$$
\tilde{A}(x, y, t)=A(t)\{1+B(x, y, t)\}
$$


where perturbed quantity $B(x, y, t)$ is written

$$
B(x, y, t)=B_{1} e^{\Omega t+i k(x \cos \theta+y \sin \theta)}+B_{2} e^{\Omega^{*} t-i k(x \cos \theta+y \sin \theta)} .
$$

Here $B_{i}, \Omega, k$ and $\theta$ are a complex constant, a growth rate with a complex quantity, the wavenumber and propagation direction of the modulational wave, respectively. Also superscript $*$ means complex conjugate.

THEOREM 1. We assume that there is the innumerable perturbed quantity, presented by equation (36), which satisfies $0<\underline{c} \leq\left|\frac{B_{2}}{B_{1}}\right| \leq \bar{c}$ as $B_{1} \rightarrow 0$. Then the sufficient condition for the stability of the uniform solution of equation (33) is

$$
k^{2} \geq \frac{A_{0}^{4}\left\{4 c_{3}\left(c \cos ^{2} \theta+d \sin ^{2} \theta\right)-c_{2}^{2}(a \cos \theta+b \sin \theta)^{2}\right\}}{\left(c \cos ^{2} \theta+d \sin ^{2} \theta\right)^{2}} \epsilon^{2} .
$$

Proof. Substituting equation (35) into equation (33) yields

$$
\begin{aligned}
& i\left[\frac{\partial A}{\partial t}(1+B)+A \frac{\partial B}{\partial t}+\left(\omega^{\prime}+\epsilon c_{1} A_{0}^{2}|1+B|^{2}\right)\left(a A \frac{\partial B}{\partial x}+b A \frac{\partial B}{\partial y}\right)+\epsilon c_{2} A^{2}(1+B)^{2}\right. \\
& \left.\quad \times\left(a A^{*} \frac{\partial B^{*}}{\partial x}+b A^{*} \frac{\partial B^{*}}{\partial y}\right)\right]+c A \frac{\partial^{2} B}{\partial x^{2}}+d A \frac{\partial^{2} B}{\partial y^{2}}+\epsilon^{2} c_{3} A_{0}^{4}|1+B|^{4} A(1+B)=0 .
\end{aligned}
$$

Using the relation

$$
\frac{\partial A}{\partial t}(1+B)=i \epsilon^{2} c_{3} A_{0}^{4} A(1+B)
$$

then it follows that eqation (38) is rewitten

$$
\begin{aligned}
& i\left(\frac{\partial B}{\partial t}+\left(\omega^{\prime}+\epsilon c_{1} A_{0}^{2}|1+B|^{2}\right)\left(a \frac{\partial B}{\partial x}+b \frac{\partial B}{\partial y}\right)+\epsilon c_{2} A_{0}^{2}(1+B)^{2}\left(a \frac{\partial B^{*}}{\partial x}+b \frac{\partial B^{*}}{\partial y}\right)\right) \\
& +c \frac{\partial^{2} B}{\partial x^{2}}+d \frac{\partial^{2} B}{\partial y^{2}}+\epsilon^{2} c_{3} A_{0}^{4}(1+B)\left(|1+B|^{4}-1\right)=0
\end{aligned}
$$

Substituting equation (36) into equation (40) yields

$$
\begin{aligned}
& e^{\Omega t+i k(x \cos \theta+y \sin \theta)}\left(\Theta_{11} B_{1}+\Theta_{12} B_{2}^{*}\right)+e^{\Omega^{*} t-i k(x \cos \theta+y \sin \theta)}\left(\Theta_{21} B_{1}^{*}+\Theta_{22} B_{2}\right) \\
& +\sum_{\substack{m=0,1, \ldots, 5 \\
n=0,1, \ldots, 5 \\
2 \leq m+n \leq 5}} e^{m\{\Omega t+i k(x \cos \theta+y \sin \theta)\}+n\left\{\Omega^{*} t-i k(x \cos \theta+y \sin \theta)\right\}} f_{m, n}\left(B_{1}, B_{2}\right)=0,
\end{aligned}
$$

where

$$
\begin{aligned}
& \Theta_{11}=i \Omega-k\left(w^{\prime}+\epsilon c_{1} A_{0}^{2}\right)(a \cos \theta+b \sin \theta)-k^{2}\left(c \cos ^{2} \theta+d \sin ^{2} \theta\right)+2 \epsilon^{2} c_{3} A_{0}^{4}, \\
& \Theta_{12}=-\epsilon k c_{2} A_{0}^{2}(a \cos \theta+b \sin \theta)+2 \epsilon^{2} c_{3} A_{0}^{4}, \\
& \Theta_{21}=\epsilon k c_{2} A_{0}^{2}(a \cos \theta+b \sin \theta)+2 \epsilon^{2} c_{3} A_{0}^{4}, \\
& \Theta_{22}=i \Omega^{*}+k\left(w^{\prime}+\epsilon c_{1} A_{0}^{2}\right)(a \cos \theta+b \sin \theta)-k^{2}\left(c \cos ^{2} \theta+d \sin ^{2} \theta\right)+2 \epsilon^{2} c_{3} A_{0}^{4} .
\end{aligned}
$$


and $f_{m, n}\left(B_{1}, B_{2}\right)$ is shown in Appendix B. We find that $f_{m, n}\left(B_{1}, B_{2}\right)$ is the function of $B_{1}$ and $B_{2}$, independent of $x, y$, and $t$.

Here Substituting $(x, y, t)=\left(x_{0}, y_{0}, t_{0}\right)$ into equation (41) yields

$$
\tilde{c}_{1}\left(\Theta_{11} B_{1}+\Theta_{12} B_{2}^{*}\right)+\tilde{c}_{1}^{*}\left(\Theta_{21} B_{1}^{*}+\Theta_{22} B_{2}\right)+\sum_{\substack{m=0,1, \ldots, 5 \\ n=0,1, \ldots, 5 \\ 2 \leq m+n \leq 5}} \tilde{c}_{m, n} f_{m, n}\left(B_{1}, B_{2}\right)=0
$$

where $\tilde{c}_{1}, \tilde{c}_{1}^{*}$ and $\tilde{c}_{m, n}$ are complex constants as follows:

$$
\begin{aligned}
& \tilde{c}_{1}=e^{\Omega t_{0}+i k\left(x_{0} \cos \theta+y_{0} \sin \theta\right)} \\
& \tilde{c}_{1}^{*}=e^{\Omega^{*} t_{0}-i k\left(x_{0} \cos \theta+y_{0} \sin \theta\right)} \\
& \tilde{c}_{m, n}=e^{m\left\{\Omega t_{0}+i k\left(x_{0} \cos \theta+y_{0} \sin \theta\right)\right\}+n\left\{\Omega^{*} t_{0}-i k\left(x_{0} \cos \theta+y_{0} \sin \theta\right)\right\}} .
\end{aligned}
$$

We also substitute $(x, y, t)=\left(x_{1}, y_{1}, t_{1}\right)$ into equation (41) then we have

$$
\tilde{c}_{1}^{\prime}\left(\Theta_{11} B_{1}+\Theta_{12} B_{2}^{*}\right)+\tilde{c}_{1}^{* \prime}\left(\Theta_{21} B_{1}^{*}+\Theta_{22} B_{2}\right)+\sum_{\substack{m=0,1, \ldots, 5 \\ n=0,1, \ldots, 5 \\ 2 \leq m+n \leq 5}} \tilde{c}_{m, n}^{\prime} f_{m, n}\left(B_{1}, B_{2}\right)=0,
$$

where $\tilde{c}_{1}^{\prime}, \tilde{c}_{1}^{* \prime}$ and $\tilde{c}_{m, n}^{\prime}$ are complex constants that $\left(x_{1}, y_{1}, t_{1}\right)$ is subsituted into equation (47) instead of $\left(x_{0}, y_{0}, t_{0}\right)$. We devide by $B_{1}$ in Equations (46) and (48) then we obtain

$$
\begin{aligned}
& \tilde{c}_{1}\left(\Theta_{11}+\Theta_{12} \frac{B_{2}^{*}}{B_{1}}\right)+\tilde{c}_{1}^{*} \frac{B_{1}^{*}}{B_{1}}\left(\Theta_{21}+\Theta_{22} \frac{B_{2}}{B_{1}^{*}}\right)+\sum_{\substack{m=0,1, \ldots, 5 \\
n=0,1, \ldots, 5 \\
2 \leq m+n \leq 5}} \tilde{c}_{m, n} \tilde{f}_{m, n}\left(B_{1}, B_{2}\right)=0 \\
& \tilde{c}_{1}^{\prime}\left(\Theta_{11}+\Theta_{12} \frac{B_{2}^{*}}{B_{1}}\right)+\tilde{c}_{1}^{* \prime} \frac{B_{1}^{*}}{B_{1}}\left(\Theta_{21}+\Theta_{22} \frac{B_{2}}{B_{1}^{*}}\right)+\sum_{\substack{m=0,1, \ldots, 5 \\
n=0,1, \ldots, 5 \\
2 \leq m+n \leq 5}} \tilde{c}_{m, n}^{\prime} \tilde{f}_{m, n}\left(B_{1}, B_{2}\right)=0
\end{aligned}
$$

where $\tilde{f}_{m, n}$, shown in Appendix C, is transformed from $f_{m, n}$ since we prepare later calculation.

From the assumption of the theorem, we can take the sequences of $B_{1}$ and $B_{2}$ such that

$$
\lim _{B_{1} \rightarrow 0} \frac{B_{2}^{*}}{B_{1}}=c_{b},
$$

where $c_{b} \in \mathbf{C}$, since $[\underline{\mathrm{c}}, \bar{c}]$ is compact. We immediately obtain

$$
\lim _{B_{1} \rightarrow 0} B_{2}^{*}=0, \quad \lim _{B_{1} \rightarrow 0} B_{2}=0, \quad \lim _{B_{1} \rightarrow 0} \frac{B_{2}}{B_{1}^{*}}=c_{b}^{*}, \quad \lim _{B_{1} \rightarrow 0} \frac{B_{1}^{*}}{B_{2}}=\frac{1}{c_{b}^{*}}, \quad \lim _{B_{1} \rightarrow 0} \frac{B_{1}}{B_{2}^{*}}=\frac{1}{c_{b}} .
$$


Moreover, we express $B_{1}$ as $\left|B_{1}\right| e^{i \theta_{b}}$, then $\frac{B_{1}^{*}}{B_{1}}=e^{-2 i \theta_{b}}$. There exsists an accumulation point of $\theta_{b 0}$ in $0 \leq \theta_{b}<2 \pi$ from the theorem of Weierstrass-Bolzano. Using the theorem of Weierstrass-Bolzano again, we can select a partially convergent sequence such as $\lim _{n \rightarrow \infty} \theta_{b n}=\theta_{b 0}$ from the sequences of $B_{1}$ that satisfies equation (51). Therefore,

$$
\lim _{B_{1} \rightarrow 0} \frac{B_{1}^{*}}{B_{1}}=e^{-2 i \theta_{b 0}}=c_{\theta} .
$$

Using equations (51), (52) and (53) and considering the state of $B_{1} \rightarrow 0$, we obtain the following coupled equations from equations (49) and (50):

$$
\begin{aligned}
& \tilde{c}_{1}\left(\Theta_{11}+c_{b} \Theta_{12}\right)+\tilde{c}_{1}^{*} c_{\theta}\left(\Theta_{21}+c_{b}^{*} \Theta_{22}\right)=0, \\
& \tilde{c}_{1}^{\prime}\left(\Theta_{11}+c_{b} \Theta_{12}\right)+\tilde{c}_{1}^{* \prime} c_{\theta}\left(\Theta_{21}+c_{b}^{*} \Theta_{22}\right)=0 .
\end{aligned}
$$

Combining equations (54) and (55),

$$
\left(\begin{array}{cc}
\tilde{c}_{1} & \tilde{c}_{1}^{*} c_{\theta} \\
\tilde{c}_{1}^{\prime} & \tilde{c}_{1}^{* \prime} c_{\theta}
\end{array}\right)\left(\begin{array}{c}
\Theta_{11}+c_{b} \Theta_{12} \\
\Theta_{21}+c_{b}^{*} \Theta_{22}
\end{array}\right)=\left(\begin{array}{l}
0 \\
0
\end{array}\right) .
$$

Here, we can select $\left(x_{0}, y_{0}, t_{0}\right)$ and $\left(x_{1}, y_{1}, t_{1}\right)$ such that a matrix $\left(\begin{array}{cc}\tilde{c}_{1} & \tilde{c}_{1}^{*} c_{\theta} \\ \tilde{c}_{1}^{\prime} & \tilde{c}_{1}^{* \prime} c_{\theta}\end{array}\right)$ has full rank. Therefore, from equation (56)

$$
\begin{aligned}
& \Theta_{11}+c_{b} \Theta_{12}=0, \\
& \Theta_{21}+c_{b}^{*} \Theta_{22}=0 .
\end{aligned}
$$

We finally obtain the relation

$$
\Theta_{11} \Theta_{22}^{*}-\Theta_{12} \Theta_{21}^{*}=0 .
$$

We obtain $\Omega$ by solving equation (58) as follows:

$$
\begin{aligned}
\Omega= & -i k\left(w^{\prime}+\epsilon c_{1} A_{0}^{2}\right)(a \cos \theta+b \sin \theta) \\
& \pm\left[k ^ { 2 } \left\{\epsilon^{2} A_{0}^{4}\left\{4 c_{3}\left(c \cos ^{2} \theta+d \sin ^{2} \theta\right)-c_{2}^{2}(a \cos \theta+b \sin \theta)^{2}\right\}\right.\right. \\
& \left.\left.-\left(c \cos ^{2} \theta+d \sin ^{2} \theta\right)^{2} k^{2}\right\}\right]^{\frac{1}{2}} .
\end{aligned}
$$

In order to be stable in the perturbation, the content of root of equation (59) must not be positive. This leads to the theorem.

REMARK 1. We assume that $0<\underline{\mathrm{c}} \leq\left|\frac{B_{2}}{B_{1}}\right| \leq \bar{c}$ only in this theorem. Although we don't assume the convergence of $\frac{B_{2}^{*}}{B_{1}}$, we have the convergent point $c_{b}$ determined uniquely by equation (57), which is unrelated to how to take partial sequences of $B_{1}$ and $B_{2}$.

REMARK 2. In equation (59), $\Omega$ has one or two points on the imaginary axis when equation (37) holds. However, when equation (37) doesn't hold, $\Omega$ has one stable point and one unstable point. Therefore, $\Omega$ is structurally unstable. 


\section{Appendix A.}

We consider a nonlinear spring, which characteristics is presented by

$$
F(w)=k_{b} w+b_{s} w^{2}
$$

instead of equation $(2)$, where $b_{s} \neq 0$. This characteristics is not so real in engineering. However, the result from equation (A.1) is important in mathematics. Then equation (4) becomes

$$
\begin{aligned}
& \frac{\partial^{2} w(x, y, t)}{\partial t^{2}}+\frac{E I}{\mu}\left\{\frac{\partial^{4} w(x, y, t)}{\partial x^{4}}+2 \frac{\partial^{4} w(x, y, t)}{\partial x^{2} \partial y^{2}}+\frac{\partial^{4} w(x, y, t)}{\partial y^{4}}\right\} \\
& +\frac{k_{b}}{\mu} w(x, y, t)+\frac{b_{s}}{\mu} w(x, y, t)^{2}=-g .
\end{aligned}
$$

Using the same transformation (equation (5)) and dimensionless variables (equation (8)), equation (A.2) becomes

$$
\begin{aligned}
& \frac{\partial^{2} \bar{w}}{\partial \bar{t}^{2}}+\frac{\partial^{4} \bar{w}}{\partial \bar{x}^{4}}+2 \frac{\partial^{4} \bar{w}}{\partial \bar{x}^{2} \partial \bar{y}^{2}}+\frac{\partial^{4} \bar{w}}{\partial \bar{y}^{4}} \\
& +\frac{l^{4}}{\pi^{4} E I}\left\{k_{b} \bar{w}+\frac{b_{s} A_{a}}{l} \bar{w}^{2}+\frac{2 b_{s} \mu g}{k_{b}} s\left(\frac{l}{\pi} \bar{x}, \frac{l}{\pi} \bar{y}\right) \bar{w}+\frac{b_{s} l}{A_{a}}\left(\frac{\mu g}{k_{b}} s\left(\frac{l}{\pi} \bar{x}, \frac{l}{\pi} \bar{y}\right)\right)^{2}\right\}=0
\end{aligned}
$$

Here we put $\tilde{\varepsilon}=\frac{A_{a}}{l}$ with $\tilde{\varepsilon}$ a small parameter. Using equation (10), then we have the following governing equation, which describes up to $\mathcal{O}\left(\varepsilon^{n}\right), n>1$ :

$$
\begin{aligned}
& \frac{\partial^{2} w(x, y, t)}{\partial t^{2}}+\frac{\partial^{4} w(x, y, t)}{\partial x^{4}}+2 \frac{\partial^{4} w(x, y, t)}{\partial x^{2} \partial y^{2}}+\frac{\partial^{4} w(x, y, t)}{\partial y^{4}} \\
& +p^{2} w(x, y, t)=\varepsilon w(x, y, t)^{2}, \\
& 0<x<\pi, \quad 0<y<\pi, \quad t>0,
\end{aligned}
$$

where all bars are dropped for convenience. In this case, $p^{2} w-\varepsilon w^{2}$ represents the restoring force due to the elastic foundation.

Substituting equation (14) into equation (A.4), we obtain

$$
\begin{aligned}
& \frac{\partial^{4} w_{0}}{\partial X_{0}^{4}}+2 \frac{\partial^{4} w_{0}}{\partial X_{0}^{2} \partial Y_{0}^{2}}+\frac{\partial^{4} w_{0}}{\partial Y_{0}^{4}}+\frac{\partial^{2} w_{0}}{\partial T_{0}^{2}}+p^{2} w_{0}=0 \\
& \frac{\partial^{4} w_{1}}{\partial X_{0}^{4}}+2 \frac{\partial^{4} w_{1}}{\partial X_{0}^{2} \partial Y_{0}^{2}}+\frac{\partial^{4} w_{1}}{\partial Y_{0}^{4}}+\frac{\partial^{2} w_{1}}{\partial T_{0}^{2}}+p^{2} w_{1} \\
& =-4 \frac{\partial^{4} w_{0}}{\partial X_{0}^{3} \partial X_{1}}-4 \frac{\partial^{4} w_{0}}{\partial X_{0} \partial X_{1} \partial Y_{0}^{2}}-4 \frac{\partial^{4} w_{0}}{\partial X_{0}^{2} \partial Y_{0} \partial Y_{1}} \\
& -4 \frac{\partial^{4} w_{0}}{\partial Y_{0}^{3} \partial Y_{1}}-2 \frac{\partial^{2} w_{0}}{\partial T_{0} \partial T_{1}}+w_{0}^{2}
\end{aligned}
$$




$$
\begin{aligned}
& \frac{\partial^{4} w_{2}}{\partial X_{0}^{4}}+2 \frac{\partial^{4} w_{2}}{\partial X_{0}^{2} \partial Y_{0}^{2}}+\frac{\partial^{4} w_{2}}{\partial Y_{0}^{4}}+\frac{\partial^{2} w_{2}}{\partial T_{0}^{2}}+p^{2} w_{2} \\
& =-4 \frac{\partial^{4} w_{1}}{\partial X_{0}^{3} \partial X_{1}}-4 \frac{\partial^{4} w_{1}}{\partial X_{0} \partial X_{1} \partial Y_{0}^{2}}-4 \frac{\partial^{4} w_{1}}{\partial X_{0}^{2} \partial Y_{0} \partial Y_{1}} \\
& \quad-4 \frac{\partial^{4} w_{1}}{\partial Y_{0}^{3} \partial Y_{1}}-2 \frac{\partial^{2} w_{1}}{\partial T_{0} \partial T_{1}}-6 \frac{\partial^{4} w_{0}}{\partial X_{0}^{2} \partial X_{1}^{2}}-4 \frac{\partial^{4} w_{0}}{\partial X_{0}^{3} \partial X_{2}}-8 \frac{\partial^{4} w_{0}}{\partial X_{0} \partial X_{1} \partial Y_{0} \partial Y_{1}} \\
& \quad-2 \frac{\partial^{4} w_{0}}{\partial X_{1}^{2} \partial Y_{0}^{2}}-4 \frac{\partial^{4} w_{0}}{\partial X_{0} \partial X_{2} \partial Y_{0}^{2}}-2 \frac{\partial^{4} w_{0}}{\partial X_{0}^{2} \partial Y_{1}^{2}}-4 \frac{\partial^{4} w_{0}}{\partial X_{0}^{2} \partial Y_{0} \partial Y_{2}} \\
& \quad-6 \frac{\partial^{4} w_{0}}{\partial Y_{0}^{2} \partial Y_{1}^{2}}-4 \frac{\partial^{4} w_{0}}{\partial Y_{0}^{3} \partial Y_{2}}-\frac{\partial^{2} w_{0}}{\partial T_{1}^{2}}-2 \frac{\partial^{2} w_{0}}{\partial T_{0} \partial T_{2}}+2 w_{0} w_{1}
\end{aligned}
$$

Equation (A.5) is the same of equation (15) so that we have the same dispersion relation of equation (19). Furthermore, we have the following solvability condition from equation (A.6):

$$
2 k_{w}^{3} \cos \theta_{0} \frac{\partial A}{\partial X_{1}}+2 k_{w}^{3} \sin \theta_{0} \frac{\partial A}{\partial Y_{1}}+\omega \frac{\partial A}{\partial T_{1}}=0,
$$

and we have the equation $w_{1}$ satisfies as follows:

$$
\begin{aligned}
& \frac{\partial^{4} w_{1}}{\partial X_{0}^{4}}+2 \frac{\partial^{4} w_{1}}{\partial X_{0}^{2} \partial Y_{0}^{2}}+\frac{\partial^{4} w_{1}}{\partial Y_{0}^{4}}+\frac{\partial^{2} w_{1}}{\partial T_{0}^{2}}+p^{2} w_{1} \\
& =A^{2} e^{2 i\left(k_{w} X_{0} \cos \theta_{0}+k_{w} Y_{0} \sin \theta_{0}-\omega T_{0}\right)}+|A|^{2}+\mathrm{cc} .
\end{aligned}
$$

Then the solution of equation (A.9) becomes

$$
w_{1}=\frac{A^{2}}{3\left(4 k_{w}^{4}-p^{2}\right)} e^{2 i\left(k_{w} X_{0} \cos \theta_{0}+k_{w} Y_{0} \sin \theta_{0}-\omega T_{0}\right)}+\frac{1}{p^{2}}|A|^{2}+\mathrm{cc} .
$$

Another solvability condition is obtained from equations (A.7) and (A.10) as follows:

$$
\begin{aligned}
& \left(6 k_{w}^{2} \cos ^{2} \theta_{0}+2 k_{w}^{2} \sin ^{2} \theta_{0}\right) \frac{\partial^{2} A}{\partial X_{1}^{2}}+4 i k_{w}^{3} \cos \theta_{0} \frac{\partial A}{\partial X_{2}}+4 k_{w}^{2} \sin 2 \theta_{0} \frac{\partial^{2} A}{\partial X_{1} \partial Y_{1}} \\
& +\left(6 k_{w}^{2} \sin ^{2} \theta_{0}+2 k_{w}^{2} \cos ^{2} \theta_{0}\right) \frac{\partial^{2} A}{\partial Y_{1}^{2}}+4 i k_{w}^{3} \sin \theta_{0} \frac{\partial A}{\partial Y_{2}}+2 i \omega \frac{\partial A}{\partial T_{2}}-\frac{\partial^{2} A}{\partial T_{1}^{2}}+\delta|A|^{2} A \\
& =0
\end{aligned}
$$


where $\delta=\frac{4\left(6 k_{w}^{4}-p^{2}\right)}{3 p^{2}\left(4 k_{w}^{4}-p^{2}\right)}$. Using equations (A.8), (A.11) and (24), we have

$$
\begin{aligned}
& 2 i \omega \frac{\partial A}{\partial T_{2}}+4 i k_{w}^{3}\left(\cos \theta_{0} \frac{\partial A}{\partial X_{2}}+\sin \theta_{0} \frac{\partial A}{\partial Y_{2}}\right) \\
& +\left\{\omega \omega^{\prime \prime} \cos ^{2} \theta_{0}+\frac{1}{3}\left(\omega \omega^{\prime \prime}+\omega^{\prime 2}\right) \sin ^{2} \theta_{0}\right\} \frac{\partial^{2} A}{\partial X_{1}^{2}} \\
& +\left\{\omega \omega^{\prime \prime} \sin ^{2} \theta_{0}+\frac{1}{3}\left(\omega \omega^{\prime \prime}+\omega^{\prime 2}\right) \cos ^{2} \theta_{0}\right\} \frac{\partial^{2} A}{\partial Y_{1}^{2}} \\
& +\frac{1}{3}\left(2 \omega \omega^{\prime \prime}-\omega^{\prime 2}\right) \sin 2 \theta_{0} \frac{\partial^{2} A}{\partial X_{1} \partial Y_{1}}+\delta|A|^{2} A=0 .
\end{aligned}
$$

Expressing $X_{1}, X_{2}, Y_{1}, Y_{2}$, and $T_{2}$ in terms of the original $x, y$, and $t$ variables, we finally obtain

$$
\begin{aligned}
& i\left\{\frac{\partial A}{\partial t}+\omega^{\prime}\left(\cos \theta_{0} \frac{\partial A}{\partial x}+\sin \theta_{0} \frac{\partial A}{\partial y}\right)\right\} \\
& +\frac{1}{2}\left\{\omega^{\prime \prime} \cos ^{2} \theta_{0}+\frac{1}{3}\left(\omega^{\prime \prime}+\frac{\omega^{\prime 2}}{\omega}\right) \sin ^{2} \theta_{0}\right\} \frac{\partial^{2} A}{\partial x^{2}} \\
& +\frac{1}{2}\left\{\omega^{\prime \prime} \sin ^{2} \theta_{0}+\frac{1}{3}\left(\omega^{\prime \prime}+\frac{\omega^{\prime 2}}{\omega}\right) \cos ^{2} \theta_{0}\right\} \frac{\partial^{2} A}{\partial y^{2}} \\
& +\frac{1}{6}\left(2 \omega^{\prime \prime}-\frac{\omega^{\prime 2}}{\omega}\right) \sin 2 \theta_{0} \frac{\partial^{2} A}{\partial x \partial y}+\frac{\epsilon^{2} \delta}{2 \omega}|A|^{2} A=0 .
\end{aligned}
$$

Equation (A.13) is rewritten by the transformation of equation (30) as follows:

$$
\begin{aligned}
& i\left\{\frac{\partial A}{\partial \tilde{t}}+\omega^{\prime}\left(\left(\sqrt{\beta} \cos \theta_{0}-\sqrt{\alpha} \sin \theta_{0}\right) \frac{\partial A}{\partial \tilde{x}}+\left(\sqrt{\beta} \cos \theta_{0}+\sqrt{\alpha} \sin \theta_{0}\right) \frac{\partial A}{\partial \tilde{y}}\right)\right\} \\
& +(2 \alpha \beta-\gamma \sqrt{\alpha \beta}) \frac{\partial^{2} A}{\partial \tilde{x}^{2}}+(2 \alpha \beta+\gamma \sqrt{\alpha \beta}) \frac{\partial^{2} A}{\partial \tilde{y}^{2}}+\frac{\epsilon^{2} \delta}{2 \omega}|A|^{2} A=0 .
\end{aligned}
$$

When using the symbols $a, b, c$, and $d$ in equation (32), we obtain the governing equation of the envelope surface of nearly monochromatic waves on an elastic, weakly nonlinear foundation with the characteristics of equation (3) as follows:

$$
i\left(\frac{\partial A}{\partial t}+\omega^{\prime}\left(a \frac{\partial A}{\partial x}+b \frac{\partial A}{\partial y}\right)\right)+c \frac{\partial^{2} A}{\partial x^{2}}+d \frac{\partial^{2} A}{\partial y^{2}}+\frac{\epsilon^{2} \delta}{2 \omega}|A|^{2} A=0,
$$

where $\sim$ is omitted for convenience. Equation (A.15) is a standard nonlinear Schrödinger equation with cubic nonlinearlity.

\section{Appendix B.}

$$
\begin{aligned}
f_{2,0}\left(B_{1}, B_{2}\right)= & -\epsilon c_{1} A_{0}^{2} k(a \cos \theta+b \sin \theta)\left(B_{1}+B_{2}^{*}\right) B_{1} \\
& -2 \epsilon c_{2} A_{0}^{2} k(a \cos \theta+b \sin \theta) B_{1} B_{2}^{*}+\epsilon^{2} c_{3} A_{0}^{4}\left(3 B_{1}^{2}+6 B_{1} B_{2}^{*}+B_{2}^{* 2}\right)
\end{aligned}
$$




$$
\begin{aligned}
f_{0,2}\left(B_{1}, B_{2}\right)= & \epsilon c_{1} A_{0}^{2} k(a \cos \theta+b \sin \theta)\left(B_{1}^{*}+B_{2}\right) B_{2} \\
& +2 \epsilon c_{2} A_{0}^{2} k(a \cos \theta+b \sin \theta) B_{1}^{*} B_{2}+\epsilon^{2} c_{3} A_{0}^{4}\left(B_{1}^{* 2}+6 B_{1}^{*} B_{2}+3 B_{2}^{2}\right) \\
f_{1,1}\left(B_{1}, B_{2}\right)= & \epsilon c_{1} A_{0}^{2} k(a \cos \theta+b \sin \theta)\left(\left|B_{2}\right|^{2}-\left|B_{1}\right|^{2}\right) \\
& +2 \epsilon c_{2} A_{0}^{2} k(a \cos \theta+b \sin \theta)\left(\left|B_{1}\right|^{2}-\left|B_{2}\right|^{2}\right) \\
& +\epsilon^{2} c_{3} A_{0}^{4}\left\{6\left(\left|B_{1}\right|^{2}+\left|B_{2}\right|^{2}\right)+6 B_{1} B_{2}+2 B_{1}^{*} B_{2}^{*}\right\} \\
f_{3,0}\left(B_{1}, B_{2}\right)= & -\epsilon c_{1} A_{0}^{2} k(a \cos \theta+b \sin \theta) B_{1}^{2} B_{2}^{*}-\epsilon c_{2} A_{0}^{2} k(a \cos \theta+b \sin \theta) B_{1}^{2} B_{2}^{*} \\
& +\epsilon^{2} c_{3} A_{0}^{4}\left(B_{1}^{3}+6 B_{1}^{2} B_{2}^{*}+3 B_{1} B_{2}^{* 2}\right) \\
f_{0,3}\left(B_{1}, B_{2}\right)= & \epsilon c_{1} A_{0}^{2} k(a \cos \theta+b \sin \theta) B_{1}^{*} B_{2}^{2}+\epsilon c_{2} A_{0}^{2} k(a \cos \theta+b \sin \theta) B_{1}^{*} B_{2}^{2} \\
& +\epsilon^{2} c_{3} A_{0}^{4}\left(B_{2}^{3}+6 B_{1}^{*} B_{2}^{2}+3 B_{1}^{* 2} B_{2}\right) \\
f_{2,1}\left(B_{1}, B_{2}\right)= & -\epsilon c_{1} A_{0}^{2} k(a \cos \theta+b \sin \theta) B_{1}\left|B_{1}\right|^{2} \\
& +\epsilon c_{2} A_{0}^{2} k(a \cos \theta+b \sin \theta)\left(B_{1}\left|B_{1}\right|^{2}-2 B_{1}\left|B_{2}\right|^{2}\right) \\
& +\epsilon^{2} c_{3} A_{0}^{4}\left\{\left(\left|B_{1}\right|^{2}+\left|B_{2}\right|^{2}\right)\left(6 B_{1}+2 B_{2}^{*}\right)\right. \\
& \left.\quad+4\left|B_{1}\right|^{2} B_{2}^{*}+6 B_{1}\left|B_{2}\right|^{2}+3 B_{1}^{2} B_{2}+B_{2}^{*}\left|B_{2}\right|^{2}\right\}
\end{aligned}
$$

$f_{1,2}\left(B_{1}, B_{2}\right)=\epsilon c_{1} A_{0}^{2} k(a \cos \theta+b \sin \theta) B_{2}\left|B_{2}\right|^{2}$

$$
\begin{aligned}
& -\epsilon c_{2} A_{0}^{2} k(a \cos \theta+b \sin \theta)\left(B_{2}\left|B_{2}\right|^{2}-2\left|B_{1}\right|^{2} B_{2}\right) \\
& +\epsilon^{2} c_{3} A_{0}^{4}\left\{\left(\left|B_{1}\right|^{2}+\left|B_{2}\right|^{2}\right)\left(2 B_{1}^{*}+6 B_{2}\right)\right. \\
& \left.+4 B_{1}^{*}\left|B_{2}\right|^{2}+6\left|B_{1}\right|^{2} B_{2}+3 B_{1} B_{2}^{2}+B_{1}^{*}\left|B_{1}\right|^{2}\right\}
\end{aligned}
$$

$f_{4,0}\left(B_{1}, B_{2}\right)=\epsilon^{2} c_{3} A_{0}^{4}\left\{B_{1}^{2} B_{2}^{* 2}+2\left(B_{1}^{3} B_{2}^{*}+B_{1}^{2} B_{2}^{* 2}\right)\right\}$

$f_{0,4}\left(B_{1}, B_{2}\right)=\epsilon^{2} c_{3} A_{0}^{4}\left\{B_{1}^{* 2} B_{2}^{2}+2\left(B_{1}^{* 2} B_{2}^{2}+B_{1}^{*} B_{2}^{3}\right)\right\}$

$f_{2,2}\left(B_{1}, B_{2}\right)=\epsilon^{2} c_{3} A_{0}^{4}\left\{\left(\left|B_{1}\right|^{2}+\left|B_{2}\right|^{2}\right)^{2}+\left|B_{1} B_{2}\right|^{2}\right.$

$$
\begin{aligned}
& +2\left(\left|B_{1}\right|^{2}+\left|B_{2}\right|^{2}\right)\left(2 B_{1} B_{2}+\left|B_{1}\right|^{2}+\left|B_{2}\right|^{2}\right) \\
& \left.+2 B_{2}\left(\left|B_{1}\right|^{2} B_{2}^{*}+B_{1}\left|B_{2}\right|^{2}\right)+2 B_{1}\left(\left|B_{1}\right|^{2} B_{2}+B_{1}^{*}\left|B_{2}\right|^{2}\right)\right\}
\end{aligned}
$$

$f_{3,1}\left(B_{1}, B_{2}\right)=\epsilon^{2} c_{3} A_{0}^{4}\left\{2\left(B_{1}^{2}+2 B_{1} B_{2}^{*}\right)\left(\left|B_{1}\right|^{2}+\left|B_{2}\right|^{2}\right)\right.$

$$
\left.+2\left(B_{1} B_{2}^{*}\left|B_{1}\right|^{2}+B_{1} B_{2}^{*}\left|B_{2}\right|^{2}+4 B_{1}^{2}\left|B_{2}\right|^{2}\right)\right\}
$$

$f_{1,3}\left(B_{1}, B_{2}\right)=\epsilon^{2} c_{3} A_{0}^{4}\left\{2\left(B_{2}^{2}+2 B_{1}^{*} B_{2}\right)\left(\left|B_{1}\right|^{2}+\left|B_{2}\right|^{2}\right)\right.$

$$
\left.+2\left(B_{1}^{*} B_{2}\left|B_{1}\right|^{2}+B_{1}^{*} B_{2}\left|B_{2}\right|^{2}+4\left|B_{1}\right|^{2} B_{2}^{2}\right)\right\}
$$

$f_{5,0}\left(B_{1}, B_{2}\right)=\epsilon^{2} c_{3} A_{0}^{4} B_{1}^{3} B_{2}^{* 2}$

$f_{0,5}\left(B_{1}, B_{2}\right)=\epsilon^{2} c_{3} A_{0}^{4} B_{1}^{* 2} B_{2}^{3}$

$f_{4,1}\left(B_{1}, B_{2}\right)=\epsilon^{2} c_{3} A_{0}^{4}\left\{B_{1}^{2}\left|B_{2}\right|^{2} B_{2}^{*}+2 B_{1}^{2} B_{2}^{*}\left(\left|B_{1}\right|^{2}+\left|B_{2}\right|^{2}\right)\right\}$

$f_{1,4}\left(B_{1}, B_{2}\right)=\epsilon^{2} c_{3} A_{0}^{4}\left\{\left|B_{1}\right|^{2} B_{1}^{*} B_{2}^{2}+2 B_{1}^{*} B_{2}^{2}\left(\left|B_{1}\right|^{2}+\left|B_{2}\right|^{2}\right)\right\}$

$f_{3,2}\left(B_{1}, B_{2}\right)=\epsilon^{2} c_{3} A_{0}^{4}\left\{B_{1}\left(\left|B_{1}\right|^{2}+\left|B_{2}\right|^{2}\right)^{2}+B_{1}\left|B_{1} B_{2}\right|^{2}+2 B_{1}\left|B_{2}\right|^{2}\left(\left|B_{1}\right|^{2}+\left|B_{2}\right|^{2}\right)\right\}$

$f_{2,3}\left(B_{1}, B_{2}\right)=\epsilon^{2} c_{3} A_{0}^{4}\left\{B_{2}\left(\left|B_{1}\right|^{2}+\left|B_{2}\right|^{2}\right)^{2}+B_{2}\left|B_{1} B_{2}\right|^{2}+2\left|B_{1}\right|^{2} B_{2}\left(\left|B_{1}\right|^{2}+\left|B_{2}\right|^{2}\right)\right\}$. 


\section{Appendix C.}

$$
\begin{aligned}
& \tilde{f}_{2,0}\left(B_{1}, B_{2}\right)=B_{1}\left[-\epsilon c_{1} A_{0}^{2} k(a \cos \theta+b \sin \theta)\left(1+\frac{B_{2}^{*}}{B_{1}}\right)\right. \\
& \left.-2 \epsilon c_{2} A_{0}^{2} k(a \cos \theta+b \sin \theta) \frac{B_{2}^{*}}{B_{1}}+\epsilon^{2} c_{3} A_{0}^{4}\left\{3+6 \frac{B_{2}^{*}}{B_{1}}+\left(\frac{B_{2}^{*}}{B_{1}}\right)^{2}\right\}\right] \\
& \tilde{f}_{0,2}\left(B_{1}, B_{2}\right)=\frac{B_{1}^{*}}{B_{1}} B_{2}\left\{\epsilon c_{1} A_{0}^{2} k(a \cos \theta+b \sin \theta)\left(1+\frac{B_{2}}{B_{1}^{*}}\right)\right. \\
& \left.+2 \epsilon c_{2} A_{0}^{2} k(a \cos \theta+b \sin \theta)+\epsilon^{2} c_{3} A_{0}^{4}\left(6+3 \frac{B_{2}}{B_{1}^{*}}+\frac{B_{1}^{*}}{B_{2}}\right)\right\} \\
& \tilde{f}_{1,1}\left(B_{1}, B_{2}\right)=B_{1}^{*}\left\{\epsilon c_{1} A_{0}^{2} k(a \cos \theta+b \sin \theta)\left(\frac{B_{2}^{*}}{B_{1}} \frac{B_{2}}{B_{1}^{*}}-1\right)\right. \\
& +2 \epsilon c_{2} A_{0}^{2} k(a \cos \theta+b \sin \theta) \\
& \left.\times\left(1-\frac{B_{2}^{*}}{B_{1}} \frac{B_{2}}{B_{1}^{*}}\right)+\epsilon^{2} c_{3} A_{0}^{4}\left(6+6 \frac{B_{2}}{B_{1}^{*}}+2 \frac{B_{2}^{*}}{B_{1}}+6 \frac{B_{2}^{*}}{B_{1}} \frac{B_{2}}{B_{1}^{*}}\right)\right\} \\
& \tilde{f}_{3,0}\left(B_{1}, B_{2}\right)=B_{1} B_{2}^{*}\left\{-\epsilon c_{1} A_{0}^{2} k(a \cos \theta+b \sin \theta)-\epsilon c_{2} A_{0}^{2} k(a \cos \theta+b \sin \theta)\right. \\
& \left.+\epsilon^{2} c_{3} A_{0}^{4}\left(6+\frac{B_{1}}{B_{2}^{*}}+3 \frac{B_{2}^{*}}{B_{1}}\right)\right\} \\
& \tilde{f}_{0,3}\left(B_{1}, B_{2}\right)=\frac{B_{1}^{*}}{B_{1}} B_{2}^{2}\left\{\epsilon c_{1} A_{0}^{2} k(a \cos \theta+b \sin \theta)+\epsilon c_{2} A_{0}^{2} k(a \cos \theta+b \sin \theta)\right. \\
& \left.+\epsilon^{2} c_{3} A_{0}^{4}\left(6+\frac{B_{2}}{B_{1}^{*}}+3 \frac{B_{1}^{*}}{B_{2}}\right)\right\} \\
& \tilde{f}_{2,1}\left(B_{1}, B_{2}\right)=\left|B_{2}\right|^{2}\left\{-\epsilon c_{1} A_{0}^{2} k(a \cos \theta+b \sin \theta) \frac{B_{1}}{B_{2}^{*}} \frac{B_{1}^{*}}{B_{2}}\right. \\
& +\epsilon c_{2} A_{0}^{2} k(a \cos \theta+b \sin \theta)\left(\frac{B_{1}}{B_{2}^{*}} \frac{B_{1}^{*}}{B_{2}}-2\right) \\
& \left.+\epsilon^{2} c_{3} A_{0}^{4}\left(12+6 \frac{B_{1}^{*}}{B_{2}}+3 \frac{B_{2}^{*}}{B_{1}}+3 \frac{B_{1}}{B_{2}^{*}}+6 \frac{B_{1}}{B_{2}^{*}} \frac{B_{1}^{*}}{B_{2}}\right)\right\} \\
& \tilde{f}_{1,2}\left(B_{1}, B_{2}\right)=B_{1}^{*} B_{2}\left\{\epsilon c_{1} A_{0}^{2} k(a \cos \theta+b \sin \theta) \frac{B_{2}}{B_{1}^{*}} \frac{B_{2}^{*}}{B_{1}}\right. \\
& -\epsilon c_{2} A_{0}^{2} k(a \cos \theta+b \sin \theta)\left(\frac{B_{2}}{B_{1}^{*}} \frac{B_{2}^{*}}{B_{1}}-2\right) \\
& \left.+\epsilon^{2} c_{3} A_{0}^{4}\left(12+3 \frac{B_{1}^{*}}{B_{2}}+6 \frac{B_{2}^{*}}{B_{1}}+3 \frac{B_{2}}{B_{1}^{*}}+6 \frac{B_{2}}{B_{1}^{*}} \frac{B_{2}^{*}}{B_{1}}\right)\right\} \\
& \tilde{f}_{4,0}\left(B_{1}, B_{2}\right)=B_{1} B_{2}^{* 2} \epsilon^{2} c_{3} A_{0}^{4}\left(3+2 \frac{B_{1}}{B_{2}^{*}}\right) \\
& \tilde{f}_{0,4}\left(B_{1}, B_{2}\right)=B_{1}^{*} \frac{B_{1}^{*}}{B_{1}} B_{2}^{2} \epsilon^{2} c_{3} A_{0}^{4}\left(3+2 \frac{B_{2}}{B_{1}^{*}}\right)
\end{aligned}
$$




$$
\begin{aligned}
& \tilde{f}_{2,2}\left(B_{1}, B_{2}\right)=B_{1}^{*}\left|B_{2}\right|^{2} \epsilon^{2} c_{3} A_{0}^{4}\left(11+6 \frac{B_{1}}{B_{2}^{*}}+6 \frac{B_{2}}{B_{1}^{*}}+3 \frac{B_{1}}{B_{2}^{*}} \frac{B_{1}^{*}}{B_{2}}+3 \frac{B_{2}^{*}}{B_{1}} \frac{B_{2}}{B_{1}^{*}}\right) \\
& \tilde{f}_{3,1}\left(B_{1}, B_{2}\right)=\left|B_{1}\right|^{2} B_{2}^{*} \epsilon^{2} c_{3} A_{0}^{4}\left(6+2 \frac{B_{1}}{B_{2}^{*}}+10 \frac{B_{2}}{B_{1}^{*}}+6 \frac{B_{2}}{B_{1}^{*}} \frac{B_{2}^{*}}{B_{1}}\right) \\
& \tilde{f}_{1,3}\left(B_{1}, B_{2}\right)=\frac{B_{1}^{*}}{B_{1}} B_{2}\left|B_{2}\right|^{2} \epsilon^{2} c_{3} A_{0}^{4}\left(6+2 \frac{B_{2}}{B_{1}^{*}}+10 \frac{B_{1}}{B_{2}^{*}}+6 \frac{B_{1}}{B_{2}^{*}} \frac{B_{1}^{*}}{B_{2}}\right) \\
& \tilde{f}_{5,0}\left(B_{1}, B_{2}\right)=B_{1}^{2} B_{2}^{* 2} \epsilon^{2} c_{3} A_{0}^{4} \\
& \tilde{f}_{0,5}\left(B_{1}, B_{2}\right)=\frac{B_{1}^{*}}{B_{1}} B_{2}^{4} \epsilon^{2} c_{3} A_{0}^{4} \frac{B_{1}^{*}}{B_{2}} \\
& \tilde{f}_{4,1}\left(B_{1}, B_{2}\right)=B_{1}\left|B_{1}\right|^{2} B_{2}^{*} \epsilon^{2} c_{3} A_{0}^{4}\left(2+3 \frac{B_{2}^{*}}{B_{1}} \frac{B_{2}}{B_{1}^{*}}\right) \\
& \tilde{f}_{1,4}\left(B_{1}, B_{2}\right)=\frac{B_{1}^{*}}{B_{1}} B_{2}^{2}\left|B_{2}\right|^{2} \epsilon^{2} c_{3} A_{0}^{4}\left(2+3 \frac{B_{1}^{*}}{B_{2}} \frac{B_{1}}{B_{2}^{*}}\right) \\
& \tilde{f}_{3,2}\left(B_{1}, B_{2}\right)=\left|B_{1}\right|^{2}\left|B_{2}\right|^{2} \epsilon^{2} c_{3} A_{0}^{4}\left(5+\frac{B_{1}}{B_{2}^{*}} \frac{B_{1}^{*}}{B_{2}}+3 \frac{B_{2}}{B_{1}^{*}} \frac{B_{2}^{*}}{B_{1}}\right) \\
& \tilde{f}_{2,3}\left(B_{1}, B_{2}\right)=B_{1}^{*} B_{2}\left|B_{2}\right|^{2} \epsilon^{2} c_{3} A_{0}^{4}\left(5+\frac{B_{2}^{*}}{B_{1}} \frac{B_{2}}{B_{1}^{*}}+3 \frac{B_{1}}{B_{2}^{*}} \frac{B_{1}^{*}}{B_{2}}\right) .
\end{aligned}
$$

Acknowledgment. The authors acknowledge the helpful discussions of Dr. T. Saigo, Taiwan University, Taipei, Taiwan. Some comments of the reviewers are also acknowledged.

\section{References}

[ 1 ] S.P. Timoshenko, Theory of Plates and Shells. McGraw-Hill, New York, 1940.

[ 2 ] S.P. Timoshenko and S. Woinowsky-Krieger, Theory of Plates and Shells McGraw-Hill, Singapore, 1970.

[ 3 ] A.C. Ugural, Stresses in Plates and Shells. McGraw-Hill, New York, 1981.

[4] A.W. Leissa, Vibration of Plates. NASA-Sp-160, 1969.

[5] H.N. Chu and G. Herrmann, Influence of large amplitudes on free flexural vibrations of rectangular elastic plates. Journal of Applied Mechnics, 23 (1956), 532-540.

[6 ] M.M. Hrabok and T.M. Hrudey, A review and catalog of plate bending finite elements. Computers and Structures, 19 (1984), 479-495.

[ 7 ] R.C. Averill and J.N. Reddy, Behavior of plate elements based on the first-order shear deformation theory. Engineering Computations, 7 (1990), 57-74.

[ 8 ] J.N. Reddy, An Introduction to the Finite Element Method, second edition. McGraw-Hill, New York, 1993.

[ 9 ] R. Haberman, Elementary Applied Partial Differential Equations. Prentice Hall, Englewood Cliff, NJ, 1983.

[10] Y. Goda, Numerical experiments on wave statistics with spectral simulation. Report Port Harbour Research Institute, 9 (1970), 3-57.

[11] S.K. Chakrabarti, R.H. Snider and P.H. Feldhausen, Mean length of runs of ocean waves. Journal of Geophysical Research, 79 (1974), 5665-5667.

[12] M.S. Longuet-Higgins, Statistical properties of wave groups in a random sea-state. Philosophical Transactions of the Royal Society of London, Series A, 312 (1984), 219-250.

[13] H. Washimi and T. Taniuti, Propagation of ion-acoustic solitary waves of small amplitude, Physics Review Letters, 17 (1966), 996-998.

[14] G.P. Agrawal, Fiber-Optic Communication System, second edition. Wiley, New York, 1997. 
[15] B.T. Nohara, Governing equations of envelope surface created by directional, nearly monochromatic waves. Journal of Society of Industrial and Applied Mathematics, 13 (2003), 75-86, (in Japanese).

[16] B.T. Nohara, Derivation and consideration of governing equations of the envelope surface created by directional, nearly monochromatic waves. International Journal of Nonlinear Dynamics and Chaos in Engineering Systems, 31 (2003), 375-392.

[17] B.T. Nohara, Governing equations of envelope surface created by nearly bichromatic waves, Propagating on an Elastic Plate and Their Stability. Japan Journal of Industrial and Applied Mathematics, 22 (2005), 87-109.

[18] B.T. Nohara and A. Arimoto, The stability of the governing equation of envelope surface created by nearly bichromatic waves propagating on an elastic plate. Nonlinear Analysis, 63 (2005), 2197-2208.

[19] B.T. Nohara, A. Arimoto and T. Saigo, Governing equations of envelopes created by nearly bichromatic waves and relation to the nonlinear Schrödinger equation. Chaos, Soliton and Fractals, 2006, to appear.

[20] B.T. Nohara and T. Saigo, Numerical simulations of the envelope created by nearly bichromatic waves. Proceeding on COMSOL Multiphysics Conference, Boston, USA, October 23-25, 2005, 383-386.

[21] M.A. Zarubinskaya and W.T. van Horssen, On the vibration on a simply supported square plate on a weakly nonlinear elastic fooundation. International Journal of Nonlinear Dynamics and Chaos in Engineering Systems, 40 (2005), 35-60.

[22] A.H. Nayfeh, Perturbation Methods. Wiley, New York, 2002.

[23] A.H. Nayfeh and D.T. Mook, Nonlinear Oscillations. Wiley, New York, 1979.

[24] T.B. Benjamin and J.E. Feir, The disintegration of wavetrains on deep water, Part 1, Theory. Journal of Fluid Mechnics, 27 (1967), 417-430. 
